\title{
MELHORAMENTO DO CAFEEIRO: XLII.PRODUTIVIDADE DE PROGÊNIES DERIVADAS DE HIBRIDAÇÃO DOS CULTIVARES LAURINA E MUNDO NOVO (')
}

\author{
ALCIDES CARVALHO $(2,4)$, LUIZ CARLOS FAZUOLI $(2,4)$ \\ P PAULO MAZZAFERA (3)
}

\begin{abstract}
RESUMO
O cultivar Laurina de Coffea arabica L. caracteriza-se pelo pequeno porte, folhas de dimensões reduzidas, frutos afilados na base, sementes pequenas e afiladas, pequeno rendimento e reduzida produção. Apresenta, no entanto, bebida de boa qualidade e baixo teor de cafeína nas sementes. Suas principais características são controladas pela ação de um par de alelos recessivos $/ r i r$, de acentuado efeito pleiotrópico. Devido ao atual interesse do comércio por produto de baixo teor de cafeina, iniciaram-se pesquisas tendo em vista principalmente aumentar a produtividade do 'Laurina'. Para esse fim, realizaram-se numerosas hibridaçōes de cafeeiros do 'Laurina' com os do 'Mundo Novo' (Coffea arabica) e, posteriormente, retrocruzamentos com o 'Mundo Novo'. Estudaram-se as progênies $F_{2}$ e retrocruzamentos com o 'Mundo Novo' ( $R C$ ) em Campinas, em um experimento, anotando-se as produções por oito anos consecutivos. Separaram-se algumas progênies $F_{2}$ em dois grupos, antes do plantio: normais $(L r L r, L r r r)$ e laurina (Irlr). Como testemunhas, usaram-se progênies do 'Mundo Novo' e 'Catuaí
\end{abstract}

(1) Trabalho apresentado no XXXII Congresso Brasileiro de Genética, SBPC, realizado em Curitiba, $\mathrm{PR}$, em 1986. Recebido para publicaçäo em 19 de jutho e aceito em 27 de outubro de 1988.

(2) Seção de Genética, Instituto Agronômico (IAC), Caixa Postal 28, 13001 Campinas (SP).

(3) Departamento de Fisiologia Vegetal, Instituto de Biologia, UNICAMP, Caixa Postal 6109, 13081 Campinas (SP).

(4) Com bolsa de pesquisa do CNPq. 
Amarelo' de $C$. arabica. $O$ conjunto de plantas $F_{2}$ do grupo laurina e os retrocruzamentos tiveram produção média maior do que as plantas $F_{2}$ normais, porém menor do que as testemunhas. Alguns retrocruzamentos e progênies $F_{2}$ apresentaram plantas com razoável produtividade, indicando que, através de retrocruzamentos com o 'Mundo Novo', podem-se obter novos tipos comerciais com as características morfológicas do 'Laurina'. Fizeram-se considerações sobre a melhor capacidade de combinação do 'Laurina' com algumas seleções do 'Mundo Novo'.

Termos de indexação: café, cultivares Laurina e Mundo Novo, hibridação, cafeína.

\section{INTRODUÇÃO}

O café 'Laurina' apresenta porte pequeno, ramificação densa, internódios curtos, folhas eliticas e pequenas, frutos e sementes pequenos e afilados na base (KRUG et al., 1938). Na literatura, é citado com várias denominaçōes. Já foi descrito como uma espécie distinta de Coffea e considerado como derivado de um híbrido interespecífico entre C. arabica e C. mauritiana (RAOUL, 1897). Aparentemente, é originário da ilha de Bourbon (CHEVALIER, 1947).

Análises genéticas efetuadas indicaram que as principais características do 'Laurina' são controladas por um par de alelos recessivos laurina ( $(r / r)$, de acentuado efeito pleiotrópico (KRUG et al., 1954). No Instituto Agronômico de Campinas, as introduçōes do 'Laurina' são de várias procedências, tanto do Brasil como de outros países. Análises genéticas de alguns exemplares indicaram o mesmo fator genético, não se podendo precisar, todavia, se originaram da mesma mutação ou se constituiam mutações independentes do mesmo alelo.

A característica estimulante, que faz do café uma das bebidas mais populares do mundo, deve-se à cafeína de suas sementes. A ausência deste alcalóide em sementes de algumas espécies de cafeeiros, todas oriundas de Madagáscar, ithas Mascarenhas e Arquipélago de Comores, permitiu que CHEVALIER (1947) as agrupasse em uma seção especial, denominada Mascarocoffea. Nas espécies do continente africano, o teor de cafeína é variável, podendo chegar a valores de até $4,0 \%$ em $C$. canephora e de $0,23-0,51 \%$ e $0,5-0,6 \%$ em $C$. eugenioides e C. racemosa respeetivamente (CARVALHO et at., 1983, CHARRIER \& BERTHAUD, 1975, 1985).

Com relação a $C$. arabica, verificou-se, em um estudo da variabilidade de teor de cafeina em diferentes cultivares, que o 'Laurina' apresenta um teor bem mais reduzido em suas sementes, 0,62\% (CARVALHO et al., 1961; TANGO \& CARVALHO, 1963). Os teores de cafeina nas folhas, hastes e raizes do 'Laurina' são, no entanto, semelhantes aos encontrados no 'Bourbon Vermelho', indicando que as diferenças se limitam apenas às sementes (FOBÉ \& CARVALHO, 1965). 
Teores de cafeina bem reduzides, de $0,6 \%$, e bem elevados, de até $1,9 \%$, foram encontrados no material de $C$. arabica nativo da Etiópia (CHARRIER \& BERTHAUD, 1975).

Apesar do efeito estimulante da cafeína, vem-se notando um crescente interesse pelo café descafeinado (CARDOSO, 1982; MORRIS, 1985; SYLVAIN, 1969), conseguido pelo emprego de solventes orgânicos (KATZ, 1980). A obtenção de cultivares sem cafeína seria de grande interesse, atendendo a parte desse mercado. Todavia, isso somente seria possivel com intenso trabalho de seleção para melhoria da produtividade e do produto dos cafés nativos de Madagáscar, que, apesar de desprovidos de cafeína, apresentam outras substâncias que depreciam sua qualidade (CHARRIER, 1978). Nesse sentido, realizaram-se trabalhos para a determinação de cafeína em cultivares de C. arabica (CARVALHO et al., 1961, 1983; TANGO \& TEIXEIRA, 1961), indicando haver considerável variabilidade quanto ao seu teor, o que torna possivel a seleção de plantas com menor conteúdo do alcalóide nas sementes. Notou-se, desde o início dos trabaIhos de melhoramento do cafeeiro, que o 'Laurina' possuia baixa produção e rendimento insatisfatório (relaçāo entre o peso de café cereja e o de beneficiado), não podendo, dessa forma, ser recomendado para plantio comercial (CARVALHO et al., 1961). Experimentos indicaram que, entre dez cultivares estudados, o 'Laurina' foi o menos produtivo, apresentando o pior rendimento e o menor peso de mil sementes (CARVALHO et al., 1973). Em outras pesquisas, em cinco localidades, constatou-se, novamente, a baixa produtividade do 'Laurina' (CARVALHO et al., 1961).

Em vista da sua pequena produtividade, efetuaram-se hibridaçōes em 1965, entre plantas do 'Laurina' e cafeeiros selecionados do 'Mundo Novo', a fim de tentar a obtenção de linhagens mais produtivas daquele. Avaliaram-se progênies $F_{2}$ dessas hibridações, bem como retrocruzamentos $\left(R_{1}\right)$ com plantas selecionadas do 'Mundo Novo', em 1969, em um experimento em Campinas, cujos resultados são apresentados neste trabalho.

\section{MATERIAL E MÉTODOS}

Cruzaram-se três cafeeiros 'Laurina', oriundos de diferentes introduçōes, de prefixos C 33-1, C 446-20 e C 1061-9, com cafeeiros 'Mundo Novo' de elevada produtividade, de prefixos CP 388-17-6, CP 399-9, CP 501-7, CP 386-2-4, CMP 376-4-3, CP 474-7 e CP 515-20. Plantaram-se os hibridos $F_{1}$ em campos de observação, sendo as progênies $F_{2}$ obtidas pela autopolinização artificial dos cafeeiros $F_{1}$. Em viveiro, identificaram-se os segregantes, facilmente reconhecidos, como normais e laurina. Das progênies $F_{2}, \mathrm{CH}$ 6209-1, $\mathrm{CH}$ 6207-2, $\mathrm{CH}$ 6204-2, CH 6205-1, CH 6208-1, CH 6222-2, CH 6226-3, CH 6227-1, CH 6254-3, $\mathrm{CH} 6256-1$ e $\mathrm{CH} 6257-1$, escolheram-se apenas as plantas do grupo laurina para 
serem estudadas no experimento, ao passo que das $\mathrm{F}_{2}$, de prefixo $\mathrm{CH} 6206-1$ e $\mathrm{CH}$ 6259-2, que correspondem a cruzamentos com plantas 'Mundo Novo' altamente produtivas, plantaram-se cafeeiros normais ( $L r L r, L r r)$ e laurina $(I r / r)$ colocados como itens independentes no ensaio. Também foram incluídas no experimento plantas originadas de seis retrocruzamentos $\left(R C_{1}\right)$ dos híbridos com plantas selecionadas do 'Mundo Novo', cujo porte é normal. O experimento foi plantado em Campinas, no Centro Experimental do Instituto Agronômico, com 36 tratamentos, em blocos ao acaso, parcelas de uma planta e cinco repetições. As progênies $\mathrm{CH}$ 6206-1 e CH 6259-2 entraram com oito tratamentos cada uma, sendo dois laurina e seis de plantas normais. Como testemunhas, usaram-se os cultivares Mundo Novo e Catuaí Amarelo. Os dados de produçāo, em quilogramas de frutos maduros, foram obtidos durante oito anos consecutivos. Empregou-se 0 teste Duncan, ao nivel de $5 \%$, para comparações de médias, efetuando-se uma análise das produções das plantas individuais (normais e laurina) das progênies $\mathrm{CH} 6206-2$ e $\mathrm{CH} 6259-2$ pelo teste $t$ a $5 \%$.

No campo, o aspecto vegetativo e a capacidade de produção das plantas individuais foram avaliados subjetivamente, atribuindo-se pontos de 1 para as piores a 10 para as melhores, durante dois anos consecutivos, independentemente do aspecto morfológico das plantas normais semelhantes ao 'Mundo Novo', ou 'Laurina' com seu distinto aspecto compacto.

\section{RESULTADOS E DISCUSSÃO}

Nota-se, no quadro 1 , com as produçōes totais médias, que, de modo geral, as progênies $F_{2}$ com características morfológicas do tipo normal $(N)$ apresentaram produçōes pouco menores do que algumas progênies $F_{2}$ do tipo laurina $(I r)$. Dois conjuntos de plantas derivadas de retrocruzamentos com 'Mundo Novo', do tipo normal $(\mathrm{N})$, se posicionaram logo em seguida às testemunhas.

Nota-se, também, a produtividade dos tratamentos correspondentes aos representantes de uma mesma população, mas diferindo quanto ao grupo de plantas, normal ou laurina. Assim, a progênie $F_{2} \mathrm{CH} 6206-2$ apresenta plantas dos dois tipos, ocorrendo o mesmo com a progênie $\mathrm{CH}$ 6259-2.

Para determinar se houve diferenças na produção dos grupos de plantas dessas progênies, fez-se análise das produções dos conjuntos de cafeeiros individuais - Quadro 2. Observou-se, no entanto, que dentro de cada progênie os tratamentos do tipo normal e do laurina não diferem estatisticamente quanto à produçăo, mas que, na progênie $\mathrm{CH}$ 6206-2, as plantas do tipo laurina deram produções maiores que as do tipo normal.

Procurou-se ainda agrupar as plantas de acordo com o objetivo de melhoramento para a sua obtenção e com o tipo morfológico da planta, distinguindo-se quatro grupos: plantas normais de primeiro retrocruzamento, $F_{2}$ 
QUADRO 1. Produção total média, em quilograma de frutos maduros, dos oito primeiros anos consecutivos de produção de populaçōes provenientes de híbridos dos cultivares Mundo Novo e Laurina de C. arabica, no Centro Experimental de Campinas (1)

\begin{tabular}{|c|c|c|c|c|}
\hline Progênies & $\begin{array}{l}\text { Geração de } \\
\text { seleção(2) }\end{array}$ & $\begin{array}{c}\text { Tipo } \\
\text { morfológico(3) }\end{array}$ & $\begin{array}{l}\text { Aspecto } \\
\text { vegetativo }\end{array}$ & $\begin{array}{l}\text { Produção } \\
\text { média(4) }\end{array}$ \\
\hline & & & & $\mathrm{kg}$ \\
\hline $\begin{array}{l}\mathrm{CH} 2077-2-5-47 \\
\text { (Catuai Amàrelo) }\end{array}$ & $\mathrm{T}$ & $N$ & 7,1 & $19,12 \mathrm{a}$ \\
\hline $\begin{array}{l}\text { CMP 386-2-5 } \\
\text { (Mundo Novo) }\end{array}$ & $\mathrm{T}$ & $N$ & 6,6 & $15,89 a b$ \\
\hline $\mathrm{CH} 7782$ & $\mathrm{RC}$ & $N$ & 5,8 & $14,97 \mathrm{a}-\mathrm{c}$ \\
\hline $\mathrm{CH} 7746$ & $\mathrm{RC}$ & $N$ & 5,5 & $12,69 b-d$ \\
\hline $\mathrm{CH} 6206-2$ & $\mathrm{~F} 2$ & Ir & 6,2 & $12,32 b-e$ \\
\hline $\mathrm{CH} 6256-1$ & $\mathrm{~F} 2$ & Ir & 6,2 & $12,30 b-f$ \\
\hline $\mathrm{CH} 6209-1$ & $\mathrm{~F} 2$ & Ir & 5,8 & $11,84 \mathrm{~b}-\mathrm{g}$ \\
\hline $\mathrm{CH} 6254-3$ & $\mathrm{~F} 2$ & tr & 6,0 & $11,78 \mathrm{~b}-\mathrm{g}$ \\
\hline CH 6204-2 & F2 & Ir & 5,7 & $11,74 \mathrm{~b}-\mathrm{g}$ \\
\hline $\mathrm{CH} 6206-2$ & $\mathrm{~F} 2$ & Ir & 5,4 & $11,19 b-g$ \\
\hline CH 7806 & $\mathrm{RC}$ & $\mathrm{N}$ & 5,2 & $10,74 b-g$ \\
\hline $\mathrm{CH} 6208-1$ & $\mathrm{~F} 2$ & Ir & 5,2 & $10,70 \mathrm{~b}-\mathrm{g}$ \\
\hline $\mathrm{CH} 7780$ & $\mathrm{RC}$ & $N$ & 5,6 & $10,64 b-g$ \\
\hline CH 6259-2 & $\mathrm{F} 2$ & $N$ & 6,3 & $10,61 b-g$ \\
\hline $\mathrm{CH} 6206-2$ & $\mathrm{~F} 2$ & $N$ & 6,0 & $10,50 \mathrm{~b}-\mathrm{g}$ \\
\hline CH 6206-2 & $\mathrm{F} 2$ & $N$ & 6,3 & $10,41 \mathrm{c}-\mathrm{g}$ \\
\hline $\mathrm{CH} 6257-1$ & $\mathrm{~F} 2$ & Ir & 5,3 & $10,36 \mathrm{c}-\mathrm{g}$ \\
\hline $\mathrm{CH} 6222-2$ & $\mathrm{~F} 2$ & Ir & 6,4 & $10,32 \mathrm{c}-\mathrm{g}$ \\
\hline $\mathrm{CH} 6259-2$ & $\mathrm{~F} 2$ & $N$ & 6,3 & $10,24 c-g$ \\
\hline $\mathrm{CH} 6226-3$ & $\mathrm{~F} 2$ & Ir & 5,6 & $9,80 \mathrm{c}-\mathrm{g}$ \\
\hline CH 6205-1 & $\mathrm{F} 2$ & $r$ & 6,1 & $9,58 c-g$ \\
\hline $\mathrm{CH} 6227-1$ & $\mathrm{~F} 2$ & Ir & 5,0 & $9,43 c-g$ \\
\hline $\mathrm{CH} 6259-2$ & $\mathrm{~F} 2$ & $N$ & 6,0 & $9,40 \mathrm{c}-\mathrm{g}$ \\
\hline $\mathrm{CH} 7810$ & $\mathrm{RC}$ & $N$ & 4,9 & $9,28 d-g$ \\
\hline $\mathrm{CH} 6207-2$ & $\mathrm{~F} 2$ & Ir & 5,7 & $9,20 \mathrm{~d}-\mathrm{g}$ \\
\hline $\mathrm{CH} 6206-2$ & $\mathrm{~F} 2$ & $N$ & 5,3 & $8,62 \mathrm{~d}-\mathrm{g}$ \\
\hline $\mathrm{CH} 6259-2$ & $\mathrm{~F} 2$ & Ir & 5,1 & $8,58 \mathrm{~d}-\mathrm{g}$ \\
\hline $\mathrm{CH} 6206-2$ & $\mathrm{~F} 2$ & $\mathrm{~N}$ & 6,5 & $8,33 d-g$ \\
\hline $\mathrm{CH} 6259-2$ & $\mathrm{~F} 2$ & $N$ & 5,7 & $8,02 d-g$ \\
\hline $\mathrm{CH} 6259-2$ & $\mathrm{~F} 2$ & Ir & 5,9 & $7,81 \mathrm{~d}-\mathrm{g}$ \\
\hline $\mathrm{CH}$ 6206-2 & $\mathrm{F} 2$ & $r$ & 5,2 & $7,73 \mathrm{~d}-\mathrm{g}$ \\
\hline $\mathrm{CH} 7747$ & $\mathrm{RC}$ & $\mathrm{N}$ & 5,7 & $6,97 \mathrm{e}-\mathrm{g}$ \\
\hline $\mathrm{CH} 6259-2$ & $\mathrm{~F} 2$ & $N$ & 5,9 & $6,89 e-g$ \\
\hline $\mathrm{CH} 6259-2$ & $\mathrm{~F} 2$ & $\mathrm{~N}$ & 5,4 & $6,85 \mathrm{e}-\mathrm{g}$ \\
\hline $\mathrm{CH} 6206-2$ & $\mathrm{~F} 2$ & $N$ & 5,1 & $6,64 \mathrm{fg}$ \\
\hline $\mathrm{CH} 6223-1$ & $\mathrm{~F} 2$ & Ir & 4,0 & $6,29 \mathrm{~g}$ \\
\hline
\end{tabular}

(1) Médias de cinco repetiçōes. (2) $\mathrm{RC}=$ Retrocruzamento, $\mathrm{F} 2$ = progênies obtidas por autofecundação dos $F_{1}, T=$ Testemunha. (3) $N=$ Nornal $_{1} t r=$ laurina. (4) Letras diferentes indicam significância pelo teste de Duncan a $5 \%$. 
QUADRO 2. Produção total média, em quilograma de frutos maduros, dos oito primeiros anos corisecutivos de produção das progênies $\mathrm{CH} 6206-2$ e $\mathrm{CH}$ 6259-2, no Centro Experimental de Campinas (1)

\begin{tabular}{|c|c|c|c|c|}
\hline Progênie & $\begin{array}{c}\text { Tipo } \\
\text { morfológico(2) }\end{array}$ & $\begin{array}{c}\text { Aspecto } \\
\text { vegetativo }\end{array}$ & $\begin{array}{l}\text { Produção } \\
\text { média }\end{array}$ & Média(3) \\
\hline & & & $\mathrm{kg}$ & \\
\hline \multirow{8}{*}{$\mathrm{CH} 6206^{\prime}=2$} & Ir & 6,2 & 12,32 & \multirow{4}{*}{$-11,75 a$} \\
\hline & Ir & 5,4 & 11,19 & \\
\hline & $\mathrm{N}$ & 6,0 & 10,50 & \\
\hline & $\mathrm{N}$ & 6,3 & 10,41 & \\
\hline & $\mathrm{N}$ & 5,3 & 8,62 & \multirow{4}{*}{$-8,71 b$} \\
\hline & $\mathrm{N}$ & 6,5 & 8,33 & \\
\hline & $\mathrm{N}$ & 5,2 & 7,73 & \\
\hline & $\mathrm{N}$ & 5,1 & 6,64 & \\
\hline \multicolumn{2}{|l|}{$C V \%=45,5$} & \multicolumn{3}{|c|}{$\bar{x}=10,23$} \\
\hline \multirow[t]{8}{*}{$\mathrm{CH} 6259-2$} & Ir & 5,1 & 8,58 & \multirow{8}{*}{$-8,20 a$} \\
\hline & Ir & 5,9 & 7,81 & \\
\hline & $\mathrm{N}$ & 6,3 & 10,61 & \\
\hline & $N$ & 6,3 & 10,24 & \\
\hline & $N$ & 6,0 & 9,40 & \\
\hline & $\mathrm{N}$ & 5,7 & 8,02 & \\
\hline & $\mathrm{N}$ & 5,9 & 6,89 & \\
\hline & $N$ & 5,4 & 6,85 & \\
\hline$C V \%=34,0$ & & & $\bar{x}=8,43$ & \\
\hline
\end{tabular}

(1) Médias de cinco repetiçōes. (2) $\mathrm{N}=$ normal; ir = laurina. (3) Médias por tipo morfológico, laurina ou normal. Letras iguais indicam nāo-significância pelo teste $t$ a $5 \%$.

normais, $F_{2}$ laurina e testemunhas. Pelo quadro 3 , observa-se que, de acordo com o teste $t$ a $5 \%$, confirmam-se os dados anteriores. Em comparação com o quadro 1 , nota-se que as testemunhas tiveram produção maior que os retrocrụzamentos e que estes tiveram produção igual às plantas do grupo $F_{2}$ laurina. As plantas do grupo $F_{2}$ laürina, por sua vez, apresentaram produção maior que o grupo $F_{2}$ normal.

Considerando-se a estratégia utilizada para obtenção dos grupos de plantas, retrocruzamento ou autofecundação, com teste de progênies, cada progênie poderia apresentar determinado conjunto de genótipos, com relação aos fatores em apreço. Nos retrocruzamentos, estes poderiam ser $L r L r$ e $L r r$, normais e, no $F_{2}, L r L r$, $L r l r$ e $I r r$, com proporção de $2 L r I r .1 L r L r$ para as plantas normais, a saber: 


\section{RETROCRUZAMENTO}

Híbridos $x$ 'Mundo Novo'

LrIr $\times$ LrLr

\section{HÍBRIDO-AUTOFECUNDAÇÃO}

LrIr X Lrir

$50 \% \operatorname{LrLr}$ (normal)

$25 \% \operatorname{Lr} r$ (normal)

$50 \%$ Lrir (normal)

$50 \%$ Lrir (normal)

$25 \%$ Irr (laurina)

QUADRO 3. Produção total média, em quilograma de frutos maduros, dos oito primeiros anos consecutivos de produção de progênies de cafeeiros agrupados de acordo com a estratégia de seleção e do tipo morfológico, no Centro Experimental de Campinas

Estratégia

de seleção
Tipo

morfológico(1)
Produção

$\overline{\text { Média(2) Amplitude }}$

$\mathrm{kg}$

$\mathrm{kg}$

Testemunhas(3)

Retrocruzamento(4)

Autofecundação(5)

Autofecundação(6)
$N$

$N$

Ir

N

$17,51 a$
$10,88 b$
$10,22 b$
$8,52 c$

$17,51 a$

9,60-23,17

3,44-19,55

2,65-18,30

$3,93-18,87$

(1) $N=$ normal, $t r=$ laurina. (2) Letras diferentes indicam significância pelo teste $t$ a $5 \%$. (3) Média de 10 cafeeiros. (4) Média de 30 cafeeiros. (5) Média de 60 cafeeiros. (5) Média de 80 cafeeiros.

Assim, quando se instalou o experimento com base na observação das caracteristicas morfológicas em viveiros devido à recessividade do alelo laurina, consideraram-se normais tanto as plantas heterozigotas ( $\mathrm{r} / \mathrm{r}$ ) quanto as homozigotas dominantes $(\mathrm{L} r \mathrm{~L} r)$. No entanto, a diferença entre as plantas normais do retrocruzamento e as de $F_{2}$ é que, no primeiro caso, há maior participação do genoma do 'Mundo Novo', que é altamente produtivo: além disso, apenas 50\% das plantas provavelmente possuam o alelo laurina. Possivelmente seja essa a razáo para alguns retrocruzamentos se posicionarem logo após as testemunhas quanto à produção (Quadro 1). Convém salientar que os dois retrocruzamentos de maior produção, $\mathrm{CH} 7782 \mathrm{e} \mathrm{CH} \mathrm{7746,} \mathrm{correspondem} \mathrm{a} \mathrm{híbridos} \mathrm{de} \mathrm{'Laurina'} \mathrm{com} \mathrm{o}$ cafeeiro CP 388-17-6 retrocruzados com esse mesmo cafeeiro, que é um dos mais produtivos até agora selecionados em Campinas. No que se refere às populaçōes $\mathrm{F}_{2}, \mathrm{CH} 6206-2$ e $\mathrm{CH}$ 6259-2, que contam cada uma um total de trinta 
plantas normais e dez do tipo laurina, distribuidas no experimento em dezesseis tratamentos, apenas na progênie $\mathrm{CH}$ 6206-2 notou-se diferença na produçāo média geral de cada grupo (Quadro 2). As plantas do tipo laurina de $\mathrm{CH} 6206-2$ produziram mais do que as normais; já na progênie $\mathrm{F}_{2} \mathrm{CH} 6259-2$, as normais tiveram médias praticamente iguais às do grupo laurina. As diferenças de produção das plantas do tipo laurina nesses grupos talvez estejam relacionadas com as plantas C 33-1 e C 1061-9 que entraram nesses cruzamentos com o cultivar Mundo Novo, bastante produtivo. A planta $C 33-1$, utilizada na sintese do hibrido $\mathrm{CH}$ 6206-2, havia sido selecionada pela produção, o que não ocorreu com o 'Laurina' de prefixo C 1061-9, que participou da formação de $\mathrm{CH}$ 6259-2. Talvez isso explique a diferença de produção das plantas do tipo laurina nessas populações. Pode-se também supor que a planta C 33-1 tenha apresentado maior capacidade de combinação com o cafeeiro CP 474-7, com o qual foi cruzada.

Com relação ao aspecto vegetativo, pode-se notar, pelo quadro 1 , que as testemunhas apresentaram as médias mais elevadas. As progênies $F_{2}$ com tipo laurina, embora variáveis, obtiveram média geral de 5,6 pontos; as plantas normais $F_{2}, 5,9$, e as dos retrocruzamentos, 5,5 pontos. A correlação entre as produções médias e as médias dos pontos atribuidos ao aspecto vegetativo revelou-se positiva e significativa $(r=0,58)$.

Para prosseguimento da seleção, sugerem-se novos retrocruzamentos com o 'Mundo Novo', principalmente com a planta CP 388-17-6, antes de efetuar autopolinização, para conseguir cafeeiros tipo laurina com maior número de fatores do 'Mundo Novo'. Isso deverá contribuir para reduzir os defeitos de baixo rendimento, pequeno peso de sementes e reduzida produção do 'Laurina'. No entanto, é mister que se façam extensivos esforços no sentido de avaliar os teores de cafeína nas populações segregantes desses cruzamentos.

Basicamente, as implicaçōes sobre a estratégia de melhoramento, potencial e limitaçōes do 'Laurina' são dependentes dessas avaliaçōes sobre o teor de cafeína nas populações segregantes. É do conhecimento generalizado na literatura que $o$ alelo laurina Ir recessivo na condição homozigota tem intenso efeito pleiotrópico, modificando a ramificação da planta e a forma das folhas e reduzindo-lhe a altura, a produçāo e o teor de cafeína. Esse conceito, porém, deve ser considerado com reservas.

O presente trabalho mostra a possibilidade de obter plantas com o fenótipo morfológico laurina e com produções que se aproximam daquelas de cultivares altamente produtivos: à medida que novos retrocruzamentos forem realizados, o nivel de produção provavelmente poderá ser aumentado. No entanto, falta ainda a informação básica sobre o efeito do alelo laurina em relação ao teor de cafeína nas populaçōes segregantes. Em outras palavras, nāo se sabe, ao certo, se o fenótipo morfológico laurina referido e o baixo teor de cafeína são realmente devidos a um efeito pleiotrópico do alelo laurina ou a locos distintos, porém ligados.

No caso de se tratar de um efeito pleiotrópico, é de antever a possibilidade de obtenção de cultivares com baixo teor de cafeina, porém somente com 
aspecto fenotípico laurina. Já no caso de essas características serem devidas à ligação genética, a sua intensidade, ou seja, a distância genética entre os dois locos, determinaria o número de indivíduos a serem analisados para obter recombinantes de fenótipo normal, porém de baixo teor do alcalóide. Por um lado, isso levaria à possibilidade vantajosa e desejável da obtenção de cultivares exatamente iguais aos hoje em cultivo, mas com baixo teor de cafeína; por outro lado, o recombinante altemativo (fenótipo laurina e teor de cafeína normal) teria mais uma característica disponivel no germoplasma de $C$. arabica a ser estudado como fonte de redução na altura e aspecto mais compacto de plantas.

Seja qual for a hipótese verdadeira a respeito da genética do fator laurina e o baixo teor em cafeina, o progresso do programa de melhoramento irá depender de extensivas análises quimicas desse alcalóide em plantas individuais de populações segregantes. Considerando as informaçōes obtidas no presente trabaiho e a crescente demanda do mercado por material com essas características, há estímulo para prosseguimento desse programa de melhoramento.

\section{SUMMARY}

\section{COFFEE BREEDING. XLII. YIELD OF PROGENIES FROM CROSSES OF LAURINA AND MUNDO NOVO CULTIVARS OF COFFEA ARABICA L.}

The Laurina cultivar of Coffea arabica L. has a reduced plant size, small leaves, small and pointed seeds and low yield capacity. However the seeds have a good cup quality and the desirable characteristic of low caffeine content. The Laurina phenotype is supposed to be controlled by a pair of recessive aleles Irtr, with pleiotropic effect. Due to the market interest for coffee with low caffeine content, selection work has been initiated some years ago in order to improve yield of the Laurina cultivar. Crosses and backcrosses $\left(B_{1}\right)$ were undertaken with selected plants of the Mundo Novo cultivar of $C$. arabica and the best $F_{1}$ plants were selfed. A yield trial was established in Campinas, State of São Paulo, Brazil, with two groups of $F_{2}$ plants, normal ( $L r L r, L r r)$ and Laurina (irrr) with $\mathrm{BC}_{\uparrow}$ plants, and 'Mundo Novo' and 'Catuaí Amarelo' as control cultivars. Results from the first eight consecutive harvests indicated that $\mathrm{BC}_{1}$ and $\mathrm{F}_{2}$ laurina plants had higher yields than the normal $F_{2}$ plants, however lower than that of the control cultivars. Some $\mathrm{BC}_{1}$ and $\mathrm{F}_{2}$ plants revealed high individual yield indicating that continued breeding of these progenies could result in selection of commercially acceptable Laurina progenies.

Index terms: Coffee Laurina and Mundo Novo cultivars, hybridization, caffeine.

\section{REFERÊNCIAS BIBUOGRÁFICAS}

CARDOSO, M. Descafeinado, desconhecido no Brasil, mas consumido na Europa, EUA. Rev. Comércio Café, Rio de Janeiro, 62(682):26-27, 1982. 
CARVALHO, A.; KRUG, C.A.; MENDES, J.E.T.; ANTUNES FILHO, H.; JUNQUEIRA, A.R.; ALOISI SOBRINHO, J.: ROCHA, T.R. \& MORAES, M.V. Melhoramento do cafeeiro. $X X I$. Comportamento regional de variedades, linhagens e progênies de café ao sol e à sombra. Bragantia, Campinas, 20:1045-1142, 1961.

; MONACO, L.C.; ALVES, S. \& FAZUOLI, L.C. Melhoramento do cafeeiro, XXXIII. Produtividade e outras características de vários cultivares em Monte Alegre do Sul. Bragantia, Campinas, 32(13):245-260, 1973.

; SONDAHL, M.R. \& SLOMA, C. Teor de cafeína em seleçōes de café. In: CONGRESSO BRASILEIRO DE PESQUISAS CAFEEIRAS, 10., Poços de Caidas, 1983. Anais. p.111-113.

; TANGO, J.S. \& MONACO, L.C. Genetic control of the caffeine content of coffee. Nature, London, 205:314, 1961.

CHARRIER, A. L'obtention et les caracteristiques de hibrids $F_{1}$ entre les Mascarocoffea et les Eucoffea. In: - La structure génetique des caféiers de la région Malgache (Mascarocoffea): leus relations avec les caféiers d'origine africaine (Eucoffea). Paris, ORSTOM, 1978. p.141-161. (Memoires ORSTOM, 87)

\& BERTHAUD, J. Botanical classification of coffee. In: CLIFFORD, M.N. \& WILSON, K.C., eds. Botany, biochemistry of beans and beverage. Westport, Ct., Avi Publishing, 1985. p.13-47.

\& BERTHAUD, J. Variation de la teneur en caféine dans le genre Coffea. Café, Cacao, The, 19(4):251-264, 1975.

CHEVALIER, A. In les caféiers du globe. Paris, Le Chevalier, 1947. (Encyclopedie biologique, fasc. 3 )

FOBÉ, L.A. \& CARVALHO, A. Contennus du caféine dans les differentes parties des caféiers en relation avec caractéristiques genétiques. Rio de Janeiro, $1965.1 \mathrm{p}$. (FAO. Primeira seção do grupo de trabalho técnico da produção de café e da proteção dos cafeeiros, resumo)

KATZ, S.N. Decafeination of coffee. In: ASIC COLLOQUIUM ON COFFEE 9., London, 1980. p.295-302.

KRUG, C.A.; CARVALHO, A. \& ANTUNES FILHO, H. Genética de Coffea. XXI. Hereditariedade das características de Coffea arabica L. var. laurina (Smeathman) D.C. Bragantia, Campinas, 13(21):247-255, 1954.

-; MENDES, J.E.T. \& CARVALHO, A. Taxonomia de Coffea arabica L. Campinas, Instituto Agronómico, 1938. 57p. (Boletim Técnico, 62)

MORRIS, S.B. Rapid growth for decafeinated coffee. Tea Coffee Trade Journal, 157(1):54-55, 1985.

RAOUL, E. Caféiers africains. In: - 2.ed. Paris, A. Chattamel, 1897. p.237-238.

SYLVAIN, P.G. Project on the production of coffee beans of low caffeine content. Turrialba, Inter-American Institute of Agricultural Sciences, 1969. 25p. (Mimeografado)

TANGO, J.S. \& CARVALHO, A. Teor de óleo e de cafeína em variedades de café. Bragantia, Campinas, 22:793-798, 1963.

\& TEIXEIRA, C.G. Teor de cafeina em progênies de café. Boletim da Superintendência dos Serviços de Café, 416:6-10, 1961. 